\section{(2) OPEN ACCESS}

\title{
Tobacco retail availability and cigarette and e- cigarette use among youth and adults: a scoping review
}

\author{
Nargiz Travis $\odot$,' David T Levy $\odot,{ }^{1}$ Patricia A McDaniel $\odot,{ }^{2}$ Lisa Henriksen $\oplus^{3}$
}

\begin{abstract}
- Additional supplemental material is published online only. To view, please visit the journal online (http://dx.doi. org/10.1136/tobaccocontrol2020-056376).
\end{abstract}

${ }^{1}$ Lombardi Comprehensive Cancer Center, Georgetown University, Washington, DC, USA ${ }^{2}$ Department of Social and Behavioral Sciences, University of California, San Francisco,

California, USA

${ }^{3}$ Stanford Prevention Research Center, Stanford University School of Medicine, Palo Alto, California, USA

Correspondence to Nargiz Travis, Lombardi Comprehensive Cancer Center, Georgetown University, Washington DC 20057, USA; nt526@georgetown.edu

Received 19 November 2020 Accepted 28 June 2021
D) Check for updates

(C) Author(s) (or their employer(s)) 2021. Re-use permitted under CC BY-NC. No commercial re-use. See rights and permissions. Published by BMJ.

To cite: Travis N, Levy DT, McDaniel PA, et al.

Tob Control Epub ahead of print: [please include Day

Month Year]. doi:10.1136/

tobaccocontrol-2020-056376

\begin{abstract}
Objective States and localities are formulating strategies to reduce the widespread retail availability of tobacco products. Evidence of associations between retailer density/proximity and tobacco use outcomes can help inform those strategies. We conducted a scoping review on tobacco retail availability and cigarette/ecigarette use in adults and youth, and considered variations in spatial units, measures of retailer exposure and outcomes across studies.
\end{abstract}

Methods A systematic search for studies examining the association between retailer density/proximity and youth and adult cigarette/e-cigarette use was conducted across MEDLINE (PubMed), Web of Science and Google Scholar through 27 August 2020 with no restrictions.

Results Thirty-five studies were included in our qualitative synthesis. While there were differences in neighbourhood definitions (eg, egocentric vs administrative), there is evidence for a positive association between higher retailer density in egocentric neighbourhoods around homes and current smoking in adults and adolescents. Administrative unit measures in some studies showed associations with adult current smoking, and adolescent lifetime and current smoking. Studies on tobacco outlet proximity to homes obtained mixed results. Density/proximity of tobacco outlets around schools showed no or inverse association with adolescent smoking, but suggests higher susceptibility to smoking. Evidence of an association between e-cigarette retail availability and e-cigarette use is limited due to a small number of studies.

Conclusion The current literature provides limited empirical evidence of the association between tobacco retailer availability and smoking or e-cigarette use. More research with uniform measures of environmental exposure to tobacco retailers is needed to allow for greater comparability between studies.

\section{INTRODUCTION}

Smoking is the leading preventable cause of premature deaths in the USA. ${ }^{1}$ Nevertheless, tobacco products are still widely available, with the vast majority sold through retail outlets. ${ }^{2}$ Tobacco retailer density has been linked to smoking among youth and adults. ${ }^{3}$ There are several mechanisms through which retailer density may affect smoking. Higher density may reduce the search costs of finding and purchasing goods, ${ }^{56}$ increase opportunities to purchase tobacco products, and encourage retailers to reduce cigarette prices and increase illegal sales to minors due to increased competition. ${ }^{7}$ Higher density may further support the ubiquity of smoking ${ }^{8}$ and increase environmental cues to smoke, whether through point-of-sale displays and advertising, ${ }^{9}$ or the mere presence of an outlet. ${ }^{10}$ Widespread availability also increases exposure to retail tobacco marketing and promotions, known to be risk factors for smoking initiation ${ }^{11}$ and impulse purchases. $^{1213}$

The high concentration of tobacco retailers around schools ${ }^{314}$ or in areas with a large proportion of residents younger than 18 years ${ }^{4}$ raises further concerns, as it exposes youth to high-risk environments during the ages in which the risks of initiation of tobacco use and transitions to daily use are greatest. Given the limited mobility and price sensitivity of youth, ${ }^{15} 16$ reducing retail density may be a particularly effective strategy to reduce youth smoking.

While there is a growing body of research examining the relationship between tobacco retailer availability and smoking behaviour, there has been inconsistency in the measures used, making comparisons difficult. For example, some studies have focused on tobacco retailer density, others on proximity to retailers. Similarly, some studies have focused on daily smoking, while others have examined smoking within the past 30 days. To date, five reviews have attempted to summarise the evidence on this topic. Notably, four reviews ${ }^{17-20}$ focused solely on youth and young adults and one did not differentiate between youth and adult studies. ${ }^{21} \mathrm{~A}$ meta-analysis ${ }^{17}$ examined the relationship between retailer density near adolescents' homes and schools and past 30-day smoking, and did not consider proximity to outlets. A narrative review ${ }^{18}$ included studies of retailer density and proximity with diverse smoking outcomes, but did not distinguish between exposure near schools versus homes. Systematic ${ }^{19}$ and methodological ${ }^{20}$ reviews examined studies on retailer density and proximity near schools and homes and diverse youth smoking outcomes. The conclusions emphasised fundamental challenges in study designs and measures of retailer exposure across studies. A recent methodological review ${ }^{21}$ examined studies on retailer density and proximity, focusing on the heterogeneity of exposure measures. However, it did not distinguish between youth and adult smoking outcomes, or consider results relative to spatial units or study location (eg, home, school, activity spaces) and did not report effect sizes. None of the prior reviews included studies on e-cigarette use, which has been increasing among US youth since $2011 .^{22} 23$

The aim of this scoping review is to summarise empirical evidence regarding the association 
between tobacco retailer density and proximity and the use of cigarettes and e-cigarettes by adults as well as youth. We aim to distinguish findings by population (adult vs youth), various cigarette/e-cigarette use outcomes, spatial units (egocentric buffers vs administrative units) and study locations. In addition, we highlight variations in density/proximity measures, differences in definitions of smoking/e-cigarette use outcomes and control variables used, which may help account for inconsistent findings across studies.

\section{METHODS}

\section{Literature search strategy}

A systematic literature search was conducted on 26 February 2020 across MEDLINE (PubMed), Web of Science and Google Scholar databases, with no restrictions on year of publication, language or article types. The search was updated on 27 August 2020. The first 100 hits on Google Scholar were screened as they were considered to be most relevant to the search topic. Search strings were created via the advanced search builder using text word combinations in the title or abstract relating to retail availability (ie, "retail", "sale*", "density", "proximity", "distance", "availability") and product use (ie, "smoking", "tobacco use", "cigarette*, "e-cigarette*"). A three-step selection process was applied. First, two authors (NT and DTL) independently screened titles and abstracts for eligibility. Second, full-text articles of selected abstracts were retrieved from databases and screened for exclusion criteria. Finally, references of fulltext articles were examined for additional relevant literature. Disagreements were discussed and resolved by consensus. The Preferred Reporting Items for Systematic Reviews and MetaAnalyses (PRISMA) checklist for scoping reviews is available in the online supplemental table S1.

\section{Inclusion criteria}

Empirical studies were included if they examined tobacco retail availability as an exposure variable, and individual-level cigarette or e-cigarette use as an outcome variable (ie, current smoking, ever smoking, initiation, cessation, quit attempts, relapse, as well as intentions to quit and smoking susceptibility (as they are closely related to product initiation and cessation)), with fulltext articles in English accessible online. Studies that investigated tobacco product categories that included e-cigarettes (eg, alternative tobacco products) were also included. Tobacco retail availability measures included, inter alia, those described in the PhenX Tobacco Regulatory Project Toolkit, such as density (number of retailers divided by land area or by total population) in person-centred buffers around study participants' homes, schools or daily activity spaces (ie, egocentric neighbourhoods); density in administrative units (eg, county, city, census tract); and proximity to the nearest tobacco retailer from homes, schools, daily activity spaces, or census area centroids. ${ }^{24}$

\section{Exclusion criteria}

Studies were excluded if they investigated outcomes not related to cigarette or e-cigarette use (eg, normative perception of smoking), used aggregated data to measure use prevalence, or examined associations in subpopulations rather than in the general population (eg, treatment-seeking smokers) to allow for comparability and meaningful interpretation of results. Descriptive geospatial studies that did not aim to provide effect sizes were also excluded.

\section{Data extraction}

The following information was synthesised from each study: first author, country, study design, data collection period, sample size, population, tobacco product type, measures of exposure, definitions of spatial units, covariates, tobacco use outcomes and effect sizes.

\section{Qualitative analysis}

Given the heterogeneity and limited empirical comparability of studies, a scoping review was selected as the most suitable approach to provide a broad overview of research on the relationship between retailer density/proximity and cigarette/e-cigarette use in both youth and adult populations and map the differences in measures of exposure and outcomes. In contrast to a systematic review, we included all relevant studies, without a priori attempting to synthesise them based on methodological quality.

\section{RESULTS}

We identified 553 records through the database searches and additional 11 records through manual checks of bibliographies. After removing duplicates, 379 abstracts were screened for eligibility and 296 were excluded. Full-text articles for the remaining 83 records were retrieved and thoroughly assessed for exclusion criteria. An updated literature search following the same methods was performed through 27 August 2020, and identified 34 unique publications, of which 2 were included (figure 1).

Overall, 35 studies, published between 2003 and 2019, were included in the qualitative synthesis (table 1). Most studies (19) were conducted in the USA, while others came from Canada, ${ }^{25-31}$ New Zealand, ${ }^{32}$ Finland, ${ }^{34}{ }^{35}$ Australia ${ }^{3637}$ and Scotland. ${ }^{38} 39$ The majority (29) examined cigarette use; few focused on e-cigarettes $^{3140-42}$ or on alternative/non-combustible tobacco products that included e-cigarettes. ${ }^{4344}$ Nearly half of the studies considered outcomes in adults (15), commonly aged $18+$ years, except in three international studies, ${ }^{332} 38$ where adults were defined as $15+$ or $16+$ years.

Studies of youth (20) included school-age participants in school-based studies and youth and young adults (ranging from 7 to 23 years old) in home-based and administrative unit-based studies (table 2).

Overall, person-centred density measures were employed in 8 adult $^{2526282935364546}$ and 10 youth studies. ${ }^{8} 303133374147-50$ Administrative density measures per land area appeared in five adult $^{27} 38434651$ and four youth studies, 494244 and density per population count appeared in one adult ${ }^{3}$ and five youth studies. ${ }^{92-55}$ Proximity was measured as the shortest distance

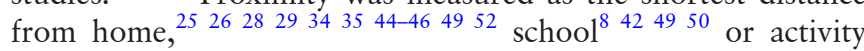
space $^{2829}$ to the nearest tobacco retailer in 15 studies, as a presence of at least one retailer per land area in four studies 8253140 and as travel time by car to the nearest retailer in one study. ${ }^{32}$

Since most studies employed multiple outcomes and measures of exposure (table 3), we grouped results for youth and adult populations by tobacco use outcomes based on the type of retailer exposure (density/proximity) and spatial units (personcentred buffers vs administrative units). Additionally, we specified the types of buffers (circular vs street network) and distances (straight-line vs roadway) used in the analyses.

\section{Retailer density and smoking outcomes in adults Current smoking}

Five cross-sectional studies investigated the relationship between tobacco retailer density and adult current smoking, defined as daily or occasional, 32936 'smoking at all nowadays' 38 and past 30-day smoking ${ }^{27}$; one cross-sectional study focused on the number of cigarettes smoked per day. ${ }^{46}$ Using person-centred 


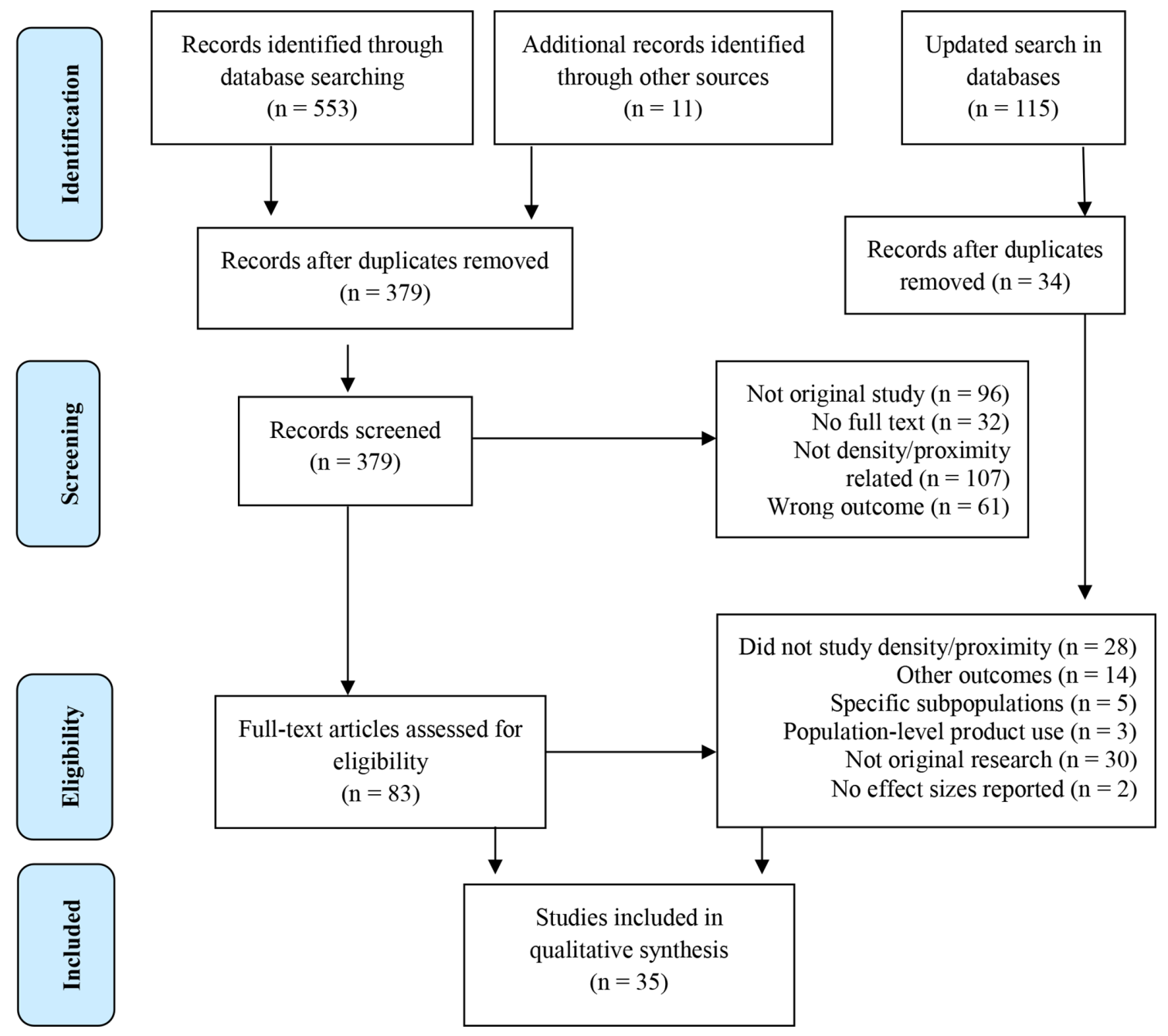

Figure 1 Preferred Reporting Items for Systematic Reviews and Meta-Analyses flow diagram of the selection process for literature review.

measures to capture density within $0.5 \mathrm{~km}$ street network buffers around participants' home address or in their daily activity spaces, higher retailer density in residential neighbourhoods was associated with current smoking in two studies from Australia ${ }^{36}$ and Canada ${ }^{29}$ with ORs ranging from 1.01 (95\% CI: 1.00 to 1.01$)^{36}$ to 1.53 (95\% CI: 1.23 to $1.91 ; \mathrm{p}<0.05),{ }^{29}$ and with a prevalence ratio $(\mathrm{PR})=1.46(95 \% \mathrm{CI}: 1.26$ to $1.70 ; \mathrm{p}<0.05)$ for density in daily activity spaces. ${ }^{29}$ Higher density derived from administrative units, such as a count per 1000 people within census tracts in Australia $^{3}$ or per square kilometre within residential ZIP codes in Scotland ${ }^{38}$ was associated with current smoking, with effect sizes ranging from $\mathrm{dy} / \mathrm{dx}$ (predicted probability) $=0.07$ (95\% CI: 0.05 to $0.10 ; \mathrm{p}<0.01)^{38}$ to $\mathrm{OR}=1.11$ (95\% CI: 1.02 to 1.21 ; $\mathrm{p}=0.018){ }^{3}$ Density per square kilometre within census tracts in a Canadian study was not associated with current smoking. ${ }^{27}$ In a US study, density within 1-mile circular buffers around homes or per square mile in corresponding census tracts was not related to the number of cigarettes smoked per day. ${ }^{46}$

\section{Smoking initiation}

In a cross-sectional US study, higher retailer density per $10 \mathrm{~km}$ of roadway within census tracts was associated with smoking initiation in young adults aged $25-34$ years (vs aged $18-24$ years) $(\mathrm{OR}=3.75,95 \% \mathrm{CI}: 1.18$ to $11.90, \mathrm{p}<0.05){ }^{43}$

\section{Smoking cessation, quit attempts and relapse}

Five studies applied person-centred density measures using circular buffers ${ }^{25}$ or street network buffers ${ }^{26283545}$ around participants' homes and investigated associations with their cessation outcomes. In two longitudinal studies, density within $500 \mathrm{~m}$ buffers was associated with reduced 30-day smoking abstinence, but only in high-poverty neighbourhoods in the USA $(\mathrm{OR}=0.94$; $95 \%$ CI: 0.90 to $0.98 ; \mathrm{p}<0.01)^{45}$ and with lower quit attempts in high-income (vs lower income) neighbourhoods $(\mathrm{OR}=0.54$; $95 \%$ CI: 0.35 to $0.85 ; \mathrm{p}<0.05)$ and increased relapse $(\mathrm{OR}=1.11$; 95\% CI: 1.00 to 1.23 ; $p>0.05$ ) in Canada. ${ }^{25}$ Smoking cessation was associated with low and intermediate levels of density within $500 \mathrm{~m}$ from homes ( $\mathrm{PR}=1.28 ; 95 \% \mathrm{CI}: 1.10$ to $1.50 ; \mathrm{p}<0.05$ ) and daily activity spaces ( $\mathrm{PR}=1.28 ; 95 \% \mathrm{CI}$ : 1.08 to 1.51 ; $\mathrm{p}<0.05$ ) in a Canadian cross-sectional study, ${ }^{28}$ and inversely related to higher availability within $500 \mathrm{~m}$ only for moderate/ heavy male smokers ( $\mathrm{PR}=0.63 ; 95 \% \mathrm{CI}: 0.49$ to $0.81 ; \mathrm{p}<0.05$ ) in a longitudinal Finnish study. ${ }^{35}$ Density within $1 \mathrm{~km}$ from home showed no associations with either 30-day abstinence or relapse in a longitudinal Canadian study. ${ }^{26}$

In two further cross-sectional studies, higher density per square kilometre or square mile within residential ZIP codes was associated with being a former (vs current) smoker in a Scottish study (dy/dx $=-0.05 ; 95 \%$ CI: -0.09 to $-0.02 ; \mathrm{p}<0.01)^{38}$ and with lower intentions to quit in the next 6 months in a US study, 


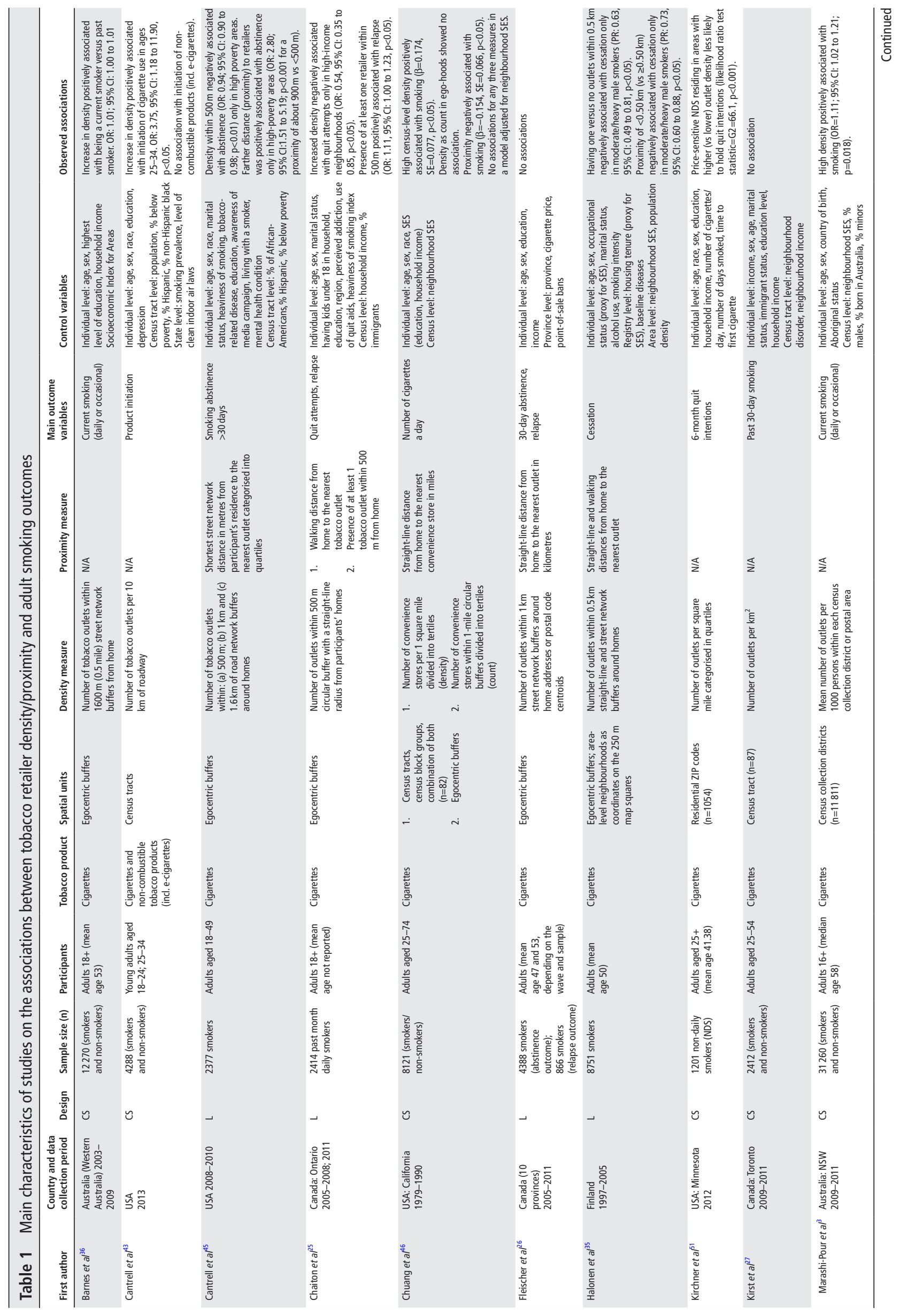




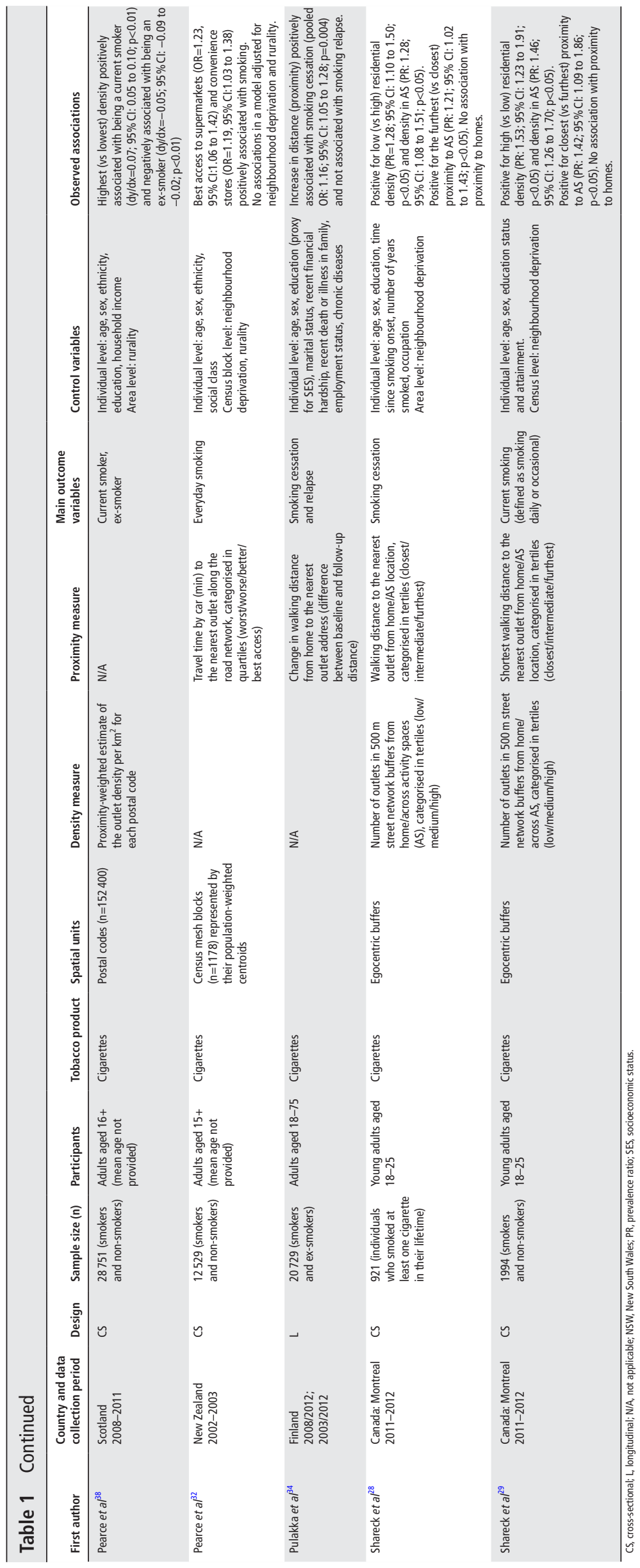




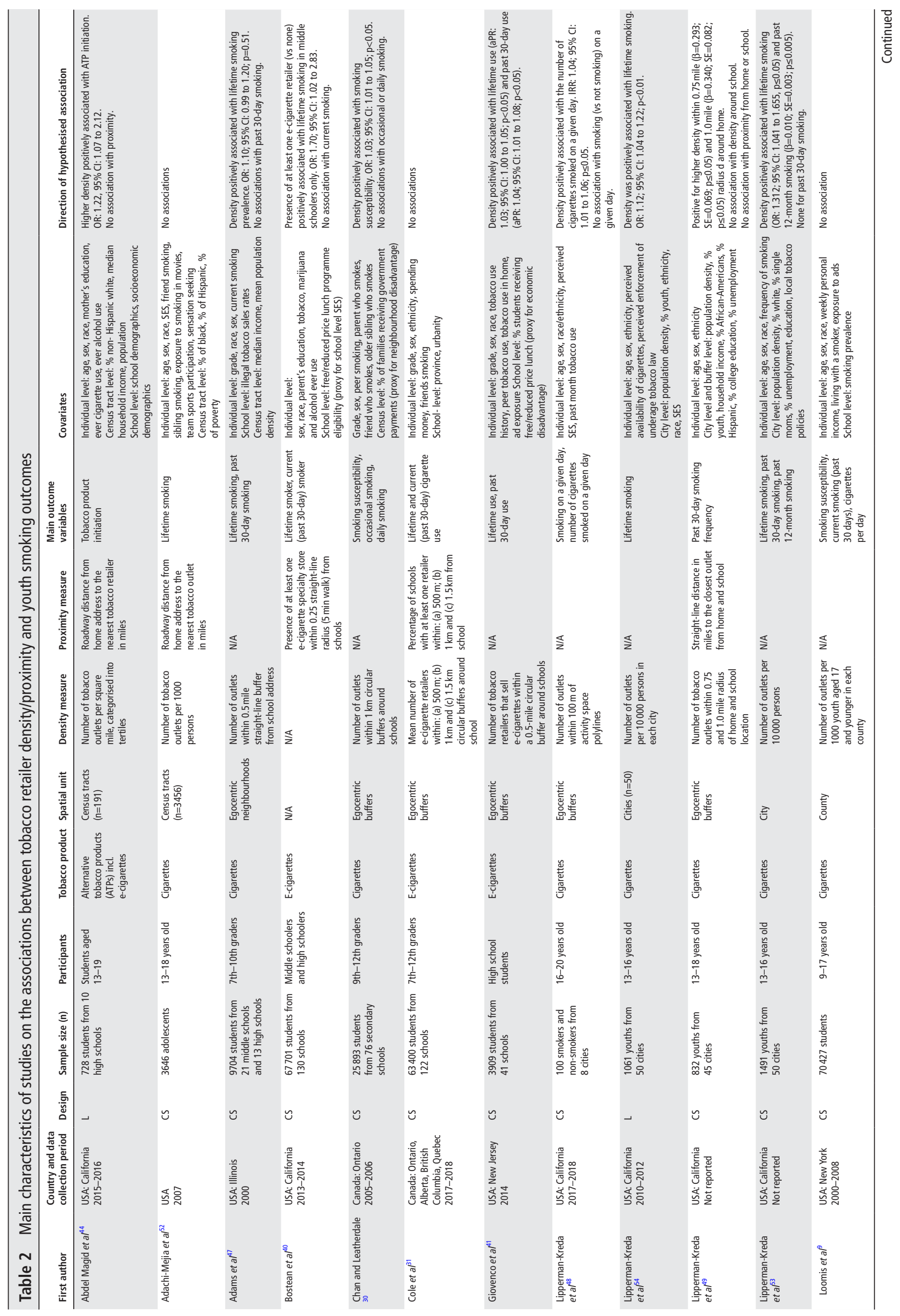




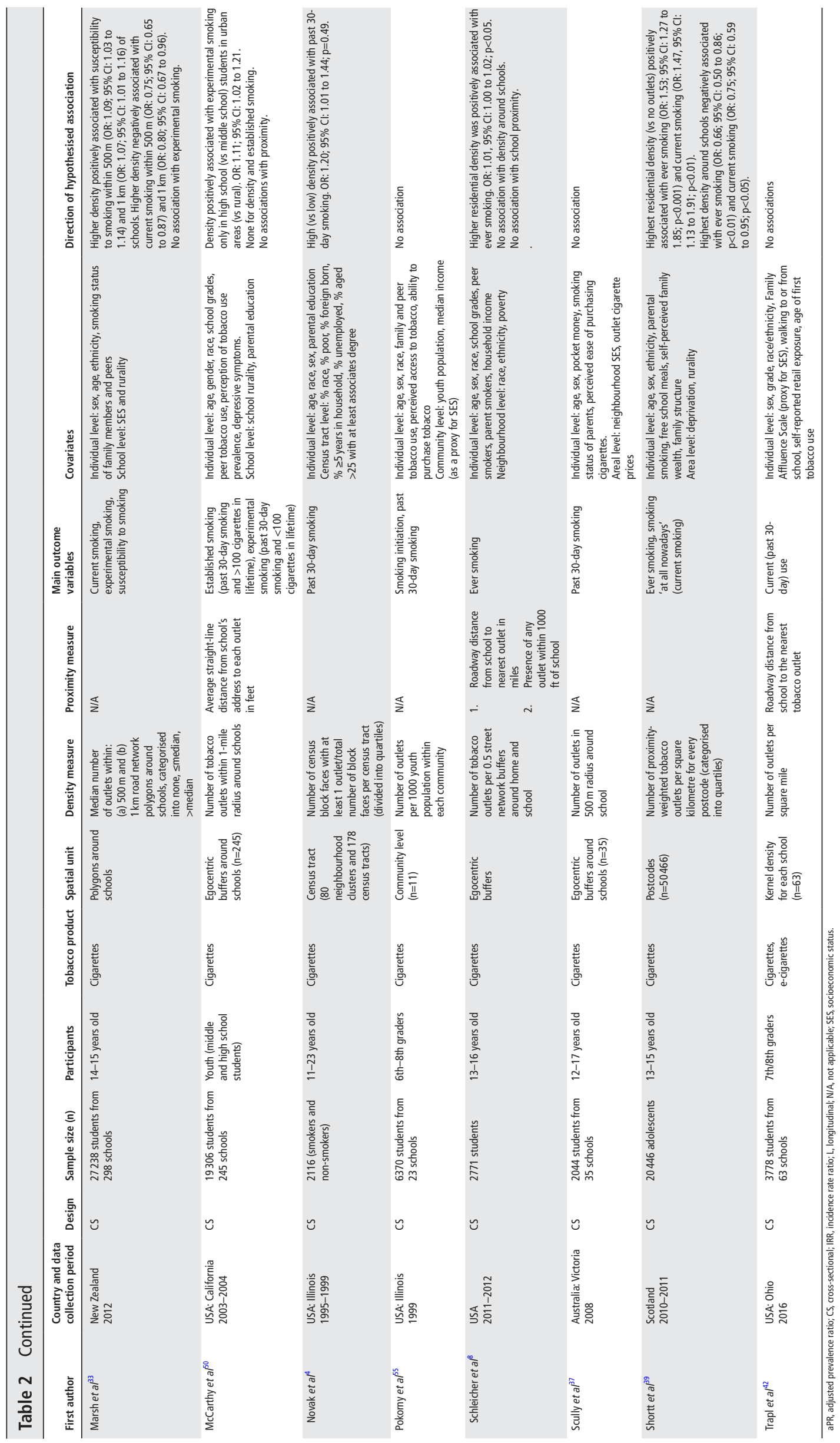




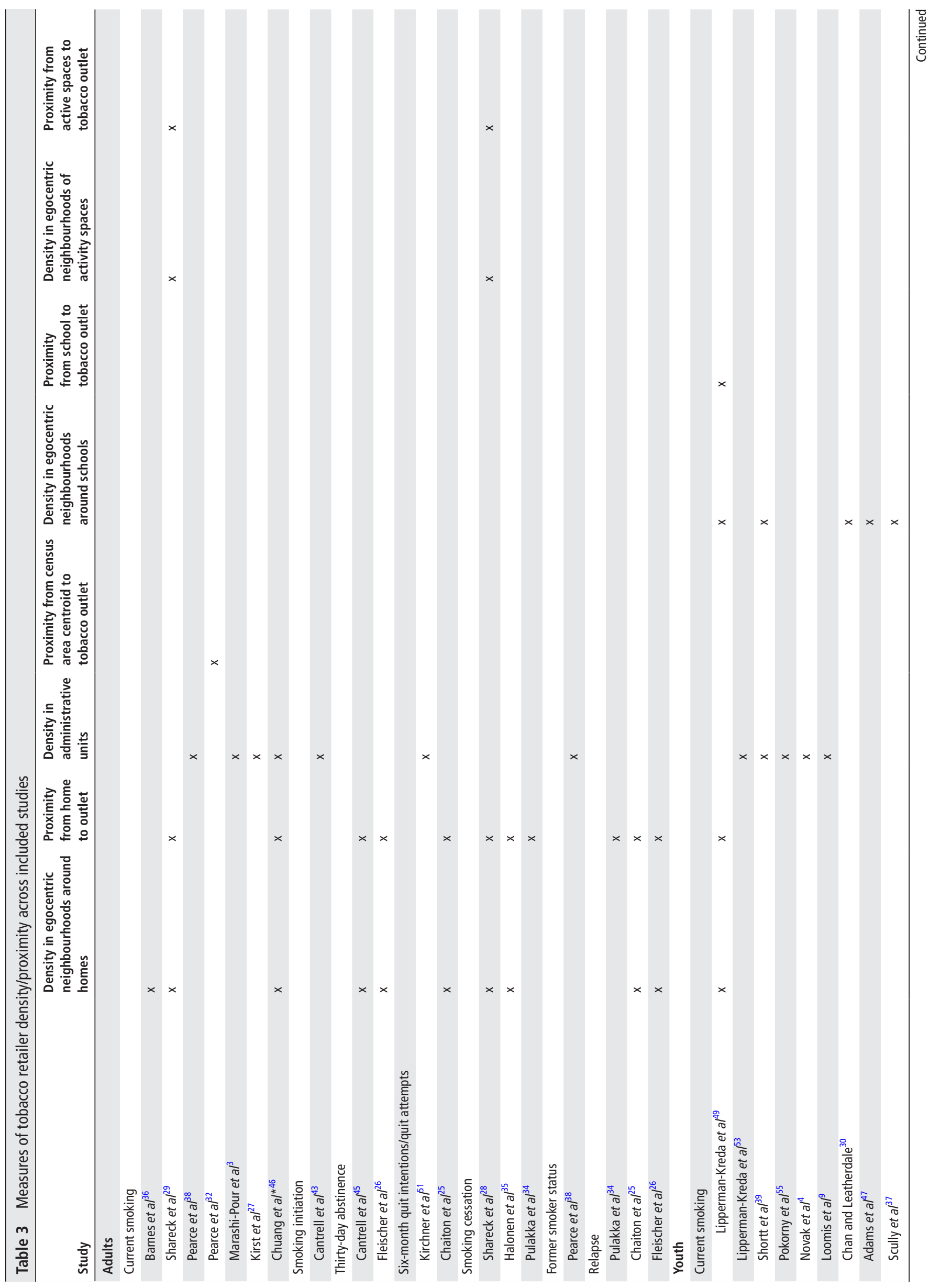




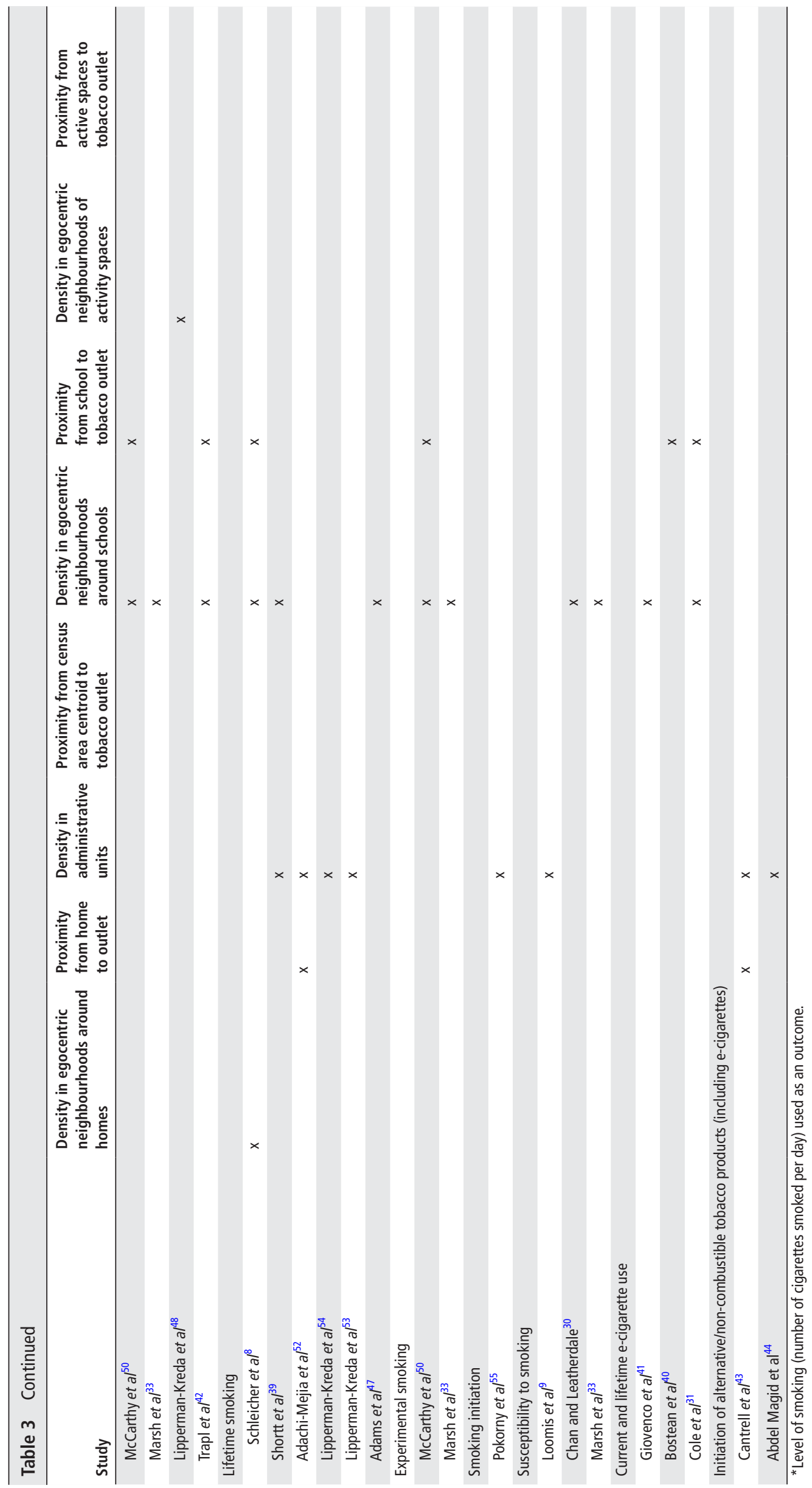


but only among price-sensitive, non-daily smokers (likelihood ratio $\mathrm{G} 2=66.1)^{51}$

\section{Proximity to tobacco retailers and smoking outcomes in adults}

Current smoking

Three cross-sectional studies investigated adult current smoking, variously defined as daily smoking, ${ }^{32}$ smoking daily or occasionally, ${ }^{29}$ and the average number of cigarettes smoked per day. ${ }^{46}$ Proximity from participants' homes to the nearest retailer, defined as the shortest walking distance (metres) in a Canadian study $^{29}$ or shortest straight-line distance (miles) in a US study, ${ }^{46}$ was not associated with current smoking or the number of cigarettes smoked per day. However, shortest walking distance to a tobacco retailer (metres) in daily activity spaces was related to current smoking in a Canadian study $(\mathrm{PR}=1.42$; 95\% CI: 1.09 to $1.86 ; \mathrm{p}<0.05) .{ }^{29}$ In New Zealand, travel time by car from census area centroids to the nearest tobacco retailer was not associated with current smoking, when adjusted for neighbourhood deprivation and rurality. ${ }^{32}$

Smoking cessation, quit attempts and relapse

Of six studies that assessed proximity from home to the nearest tobacco outlet, three measured walking distance (metres), ${ }^{25} 2845$ two measured straight-line distance (metres, kilometres) ${ }^{26} 34$ and one compared both. ${ }^{35}$ All studies but one were longitudinal. A greater walking distance was associated with higher odds of 30-day smoking abstinence in a US study, but only in highpoverty areas $(\mathrm{OR}=2.80 ; 95 \% \mathrm{CI} \text { : } 1.51 \text { to } 5.19 \text {; } \mathrm{p}<0.001)^{45}$; and was otherwise unrelated to quit attempts and relapse in one Canadian study, ${ }^{25}$ and to smoking cessation in another cross-sectional Canadian study. ${ }^{28}$ However, the same measure in daily activity spaces was associated with smoking cessation $(\mathrm{PR}=1.21 ; 95 \% \mathrm{CI}: 1.02$ to $1.43 ; \mathrm{p}<0.05) .{ }^{28}$ In studies from Finland ${ }^{34}$ and Canada, ${ }^{26}$ greater straight-line distance from home to the nearest tobacco retailer was positively associated with smoking cessation $(\mathrm{OR}=1.16 ; 95 \% \mathrm{CI}$ : 1.05 to $1.28 ; \mathrm{p}=0.004),{ }^{34}$ but not with 30 -day smoking abstinence ${ }^{26}$ or relapse. ${ }^{2634}$ In another Finnish study, smoking cessation was inversely associated with closer proximity using both measures, but only in moderate/heavy male smokers $(\mathrm{PR}=0.73$, 95\% CI: 0.60 to $0.88 ; \mathrm{p}<0.05) .{ }^{35}$

\section{Retailer density and adolescents' smoking outcomes Current smoking}

Adolescent current smoking was defined in seven cross-sectional studies as past 30-day smoking, ${ }^{4949535}$ smoking 'at all nowadays ${ }^{39}$ or 'any cigarette use on a given day'. ${ }^{48}$ All but one study $^{39}$ were conducted in the USA. Greater density within 0.75 -mile circular buffers around homes was associated with higher smoking frequency $(\beta=0.293 ; \mathrm{SE}=0.069 ; \mathrm{p} \leq 0.05) .{ }^{49}$ Density within $100 \mathrm{~m}$ of daily activity space polylines was not associated with youth smoking in a study that used real-time geographical ecological momentary assessment. ${ }^{48}$ While density per square kilometre within residential ZIP $\operatorname{codes}^{39}$ and within census tracts ${ }^{4}$ was positively associated with increased smoking, with ORs ranging from 1.20 (95\% CI: 1.01 to 1.44$)$ to 1.47 (95\% CI: 1.13 to $1.91 ; \mathrm{p}<0.01$ ), larger administrative measures, such as county-level density per 1000 people (aged 17 years and younger) ${ }^{955}$ and city-level density per 10000 people, ${ }^{53}$ showed no associations.

\section{Lifetime smoking}

Adolescent lifetime smoking was defined in five studies as ever smoking a cigarette, ${ }^{39} 52$ ever trying a cigarette (even one puff) ${ }^{8}$ and ever smoking a whole cigarette (more than just a few puffs). ${ }^{53}{ }^{54}$ Most studies were cross-sectional and conducted in the USA, except for one longitudinal study ${ }^{54}$ and one conducted in Scotland. ${ }^{39}$ Higher retailer density within 0.5 mile of egocentric road network buffers around homes was associated with higher odds of lifetime smoking $(\mathrm{OR}=1.01$; 95\% $\mathrm{CI}$ : 1.00 to $1.02 ; \mathrm{p}<0.05){ }^{8}$ Administrative measures, such as density per square kilometre in residential ZIP codes $(\mathrm{OR}=1.53$; 95\% CI: 1.27 to $1.85 ; \mathrm{p}<0.001)^{39}$ and density per 10000 population in cities $(\mathrm{OR}=1.12 ; 95 \% \mathrm{CI}: 1.04$ to $1.22 ; \mathrm{p}<0.01$ and $\mathrm{OR}=1.312$; $95 \% \mathrm{CI}$ : 1.041 to $1.655 ; \mathrm{p} \leq 0.05)^{53} 54$ also correlated with lifetime smoking, while nationwide density per 1000 persons showed no associations. ${ }^{52}$

\section{Smoking initiation and susceptibility}

In two cross-sectional US studies, adolescents' smoking initiation $^{55}$ and susceptibility to smoking ${ }^{9}$ were not associated with retailer density per 1000 people (aged 17 years and younger) within a county or community.

\section{School-level retailer density and adolescents' smoking outcomes \\ Current smoking}

Eight cross-sectional studies considered adolescent current smoking, defined as past 30-day smoking, ${ }^{37424749}$ past 30-day smoking and more than 100 cigarettes in a lifetime, ${ }^{3350}$ occasional or daily smoking, ${ }^{30}$ or smoking 'at all nowadays'. ${ }^{39}$ Smoking was not associated with higher retailer density in egocentric buffers around schools in three US, one Canadian (Ontario) and one Australian (Victoria) studies, ${ }^{30} 37474950$ and inversely associated with higher density within $500 \mathrm{~m}$ road network buffers in one New Zealand study $(\mathrm{OR}=0.75 ; 95 \% \mathrm{CI}$ : 0.65 to 0.87 ; $\mathrm{p}<0.05){ }^{33}$ An administrative measure of density per square mile around schools in the USA showed no association, ${ }^{42}$ while density per square kilometre within school ZIP codes in a Scottish study $(\mathrm{OR}=0.75 ; 95 \% \mathrm{CI}: 0.59 \text { to } 0.95 ; \mathrm{p}<0.05)^{39}$ showed an inverse relationship.

\section{Lifetime and experimental smoking}

Five cross-sectional studies considered adolescent lifetime smoking, defined as ever smoking a cigarette ${ }^{39} 47$ or ever trying a cigarette (even one puff), ${ }^{8}$ or experimental smoking, defined as past 30-day smoking and having smoked less than 100 cigarettes in a lifetime. ${ }^{33}{ }^{50}$ In two US studies, higher densities within 0.5 mile and 1-mile circular buffers around schools were associated with adolescent lifetime smoking $(\mathrm{OR}=1.10$; 95\% CI: 0.99 to $1.20 ; \mathrm{p}=0.51),{ }^{47}$ and with experimental smoking $(\mathrm{OR}=1.11$; 95\% CI: 1.02 to 1.21 ) only for high school students in urban areas. ${ }^{50}$ Density within 0.5 -mile, 500-metre and 1-kilometre road network buffers around schools showed no association with lifetime smoking in the USA ${ }^{8}$ or experimental smoking in New Zealand. ${ }^{33}$ In one Scottish study, higher density per square kilometre within schools' ZIP codes was inversely associated with lifetime smoking $(\mathrm{OR}=0.66 ; 95 \% \mathrm{CI}$ : 0.50 to $0.86 ; \mathrm{p}<0.01) .{ }^{39}$

\section{Susceptibility to smoking}

Susceptibility to smoking (intention to try a cigarette soon or in the next year or if offered to try by a best friend) was associated with higher density within $1 \mathrm{~km}$ circular buffers in a cross-sectional Ontario study $(\mathrm{OR}=1.03$; $95 \% \mathrm{CI}$ : 1.01 to 1.05 ; 
$\mathrm{p}<0.05)^{30}$ and within $1 \mathrm{~km}$ road network buffers around schools in a cross-sectional New Zealand study $(\mathrm{OR}=1.07$; 95\% CI: 1.01 to $1.16 ; \mathrm{p}<0.05)$. $^{33}$

\section{Retailer proximity to homes and adolescents' smoking outcomes}

Current and lifetime smoking

In two cross-sectional US studies, past 30-day and lifetime smoking was not associated with proximity to the closest retailer from home, measured either as a straight-line distance ${ }^{49}$ or distance in roadway miles. ${ }^{52}$

\section{Retailer proximity to schools and adolescents' smoking outcomes}

\section{Current smoking}

Three cross-sectional US studies examined current adolescent smoking, defined as past 30 -day smoking ${ }^{42} 49$ or past 30 -day smoking and more than 100 lifetime cigarettes ${ }^{50}$ and retailer proximity to schools, measured as a straight-line distance in feet $^{50}$ or in miles ${ }^{49}$ and street network distance. ${ }^{42}$ None found significant associations.

\section{Lifetime and experimental smoking}

Two cross-sectional US studies explored the relationship between retailer proximity to adolescents' schools, defined both as a distance in roadway miles, and the presence of at least one outlet within $1000 \mathrm{ft}^{8}$, or as a straight-line distance, ${ }^{50}$ and lifetime smoking or experimental smoking. Neither found an association.

\section{E-cigarette retailer density/proximity and e-cigarette use}

Four cross-sectional studies investigated the density of e-cigarette retailers near schools and adolescent lifetime and/or current (past 30-day) use. In a US study, a count of tobacco retailers that sold e-cigarettes within a 0.5 -mile circular buffers around schools was associated with current use (adjusted PR $(\mathrm{aPR})=1.04 ; 95 \% \mathrm{CI}: 1.01$ to $1.08 ; \mathrm{p}<0.05)$ and lifetime use $(\mathrm{aPR}=1.03 ; 95 \% \mathrm{CI}: 1.00$ to $1.05 ; \mathrm{p}<0.05) .{ }^{41}$ However, the number of vape shops within $0.5,1.0$ and $1.5 \mathrm{~km}$ circular buffers was not associated with current or lifetime use in a Canadian study. ${ }^{31}$ In a US study, the number of e-cigarette retailers per square mile within schools' ZIP codes was not related to current use among students. ${ }^{42}$ Proximity, defined as a presence of at least one e-cigarette specialty store within a 0.25 -mile buffers from schools, was only associated with lifetime use in middle school students (vs high school students) (OR $=1.70 ; 95 \% \mathrm{CI}$ : 1.02 to 2.83 ) and not associated with current use. ${ }^{40}$ In a Canadian study, the presence of at least one e-cigarette retailer within $0.5,1.0$ and $1.5 \mathrm{~km}$ circular buffers around schools was not associated with lifetime or current use. ${ }^{31}$ In a US study, walking distance from school to the closest e-cigarette retailer was not associated with students' current e-cigarette use. ${ }^{42}$

While no studies examined the initiation of e-cigarettes (exclusively), two considered initiation of alternative/noncombustible tobacco products (including e-cigarettes) among youth and young adults. A longitudinal study in the USA showed that living in census tracts with higher tobacco retailer density per square mile was positively associated with adolescents' initiation of alternative tobacco products $(\mathrm{OR}=1.22,95 \% \mathrm{CI}: 1.07$ to 2.12), but no association was found for retailer proximity from home measured in roadway miles. ${ }^{44}$ In a cross-sectional US study, living in tracts with higher tobacco retailer density (count per $10 \mathrm{~km}$ of roadway) was not associated with non-combustible product initiation in young adults. ${ }^{43}$

\section{DISCUSSION}

Our scoping review summarises evidence on the association between tobacco retailer availability and the use of cigarettes and e-cigarettes in adults and adolescents, while considering variations in tobacco use outcomes and measures of density/ proximity.

For adults, evidence from cross-sectional research showed a positive association between current smoking and both personcentred measures around homes (two of two studies) ${ }^{29} 36$ or in daily activity spaces (one of one) ${ }^{29}$ and administrative units (two of three) ( $38^{38}$ of retailer density. Evidence on the relationship between current smoking and retailer proximity to homes, daily activity spaces or administrative unit centroids was more limited (one of three). ${ }^{29}$ There was also evidence, mainly from longitudinal studies, of associations between higher personcentred density near homes and lower smoking cessation (two of two), ${ }^{28} 35$ quit attempts (one of one), ${ }^{25} 30$-day abstinence (one of two $)^{45}$ and higher relapse (one of two). ${ }^{25}$ However, these associations were usually limited to specific populations, such as price-sensitive non-daily smokers, ${ }^{51}$ moderate/heavy male smokers, ${ }^{35}$ or residents of high-poverty ${ }^{45}$ or high-income neighbourhoods. ${ }^{25}$ Farther retailer proximity from homes showed associations with higher cessation (two of three), ${ }^{34} 35$ but was not related to smoking relapse (none of three).

For adolescents, evidence gathered from predominantly crosssectional research indicated a positive association of personcentred measures of retailer density near homes and daily activity spaces with current smoking and the number of cigarettes smoked (two of two), ${ }^{48}$ as well as lifetime smoking (one of one). ${ }^{8}$ For administrative units, there was some evidence of a positive association with density and current smoking (two of five), ${ }^{49}$ but evidence for lifetime smoking was more consistent (three of four). ${ }^{395354}$ Higher density near schools showed no or inverse association with adolescent current smoking, but was related to greater susceptibility to smoke (two of two). ${ }^{3033}$ There was no evidence that retailer proximity to homes or schools was related to adolescent smoking.

Given e-cigarettes' popularity among youth, research on association of use with retail density/proximity of e-cigarettes is surprisingly scarce. Existing studies focused on e-cigarette retailer availability near schools and suggest that adolescent current e-cigarette may be related to retailer density (one of three), ${ }^{41}$ but not proximity (none of three). Inadequate data about which tobacco retailers sell e-cigarettes are an obstacle to research on this topic. Studies of vape shops (that sell e-cigarettes exclusively) may underestimate retail availability of e-cigarettes, while studies of all tobacco retailers surely overestimate it.

Our findings are consistent with a meta-analysis that found a small but significant positive relationship between tobacco retailer density around adolescents' homes (but not schools) and past month smoking. ${ }^{17}$ While results of a narrative review ${ }^{18}$ were inconclusive due to heterogeneity and small number of included studies, systematic ${ }^{19}$ and methodological ${ }^{20}$ reviews also found some support for a positive association of youth smoking with higher retailer density around homes, but not with proximity to homes or schools. A recent methodological review ${ }^{21}$ concluded that there was an overall positive relationship between tobacco retailer density and smoking prevalence and initiation, with retailer proximity inversely related to smoking cessation. However, these findings did not distinguish between adult and youth smoking outcomes or the location of retailer exposure, thus limiting comparability of included studies and a meaningful interpretation of results. In contrast, our review provides 
a more comprehensive analysis, highlighting that while tobacco retailer density/proximity around homes and in activity spaces is related to both adolescent and adult smoking, retailer availability around schools is not (or inversely) related to adolescent smoking prevalence, but rather to susceptibility to smoking and cigarette experimentation.

Variation in measurements of retailer density/proximity across studies may partially explain the inconsistent evidence, since inaccurate definition of neighbourhoods contributes to spatial misclassification of exposure. Administrative definitions of neighbourhoods are more common and convenient, but assuming the same exposure for all individuals may mask true associations. Egocentric definitions of neighbourhoods or activity spaces are optimal to estimate individual-level retailer exposures, but the data are more difficult to obtain. Although circular buffers are more commonly used to define egocentric neighbourhoods, street network buffers better reflect real-life settings since they account for physical barriers. ${ }^{56}$ Similarly, roadway distance or travel time is a more appropriate measure of proximity as opposed to straight-line distance, ${ }^{35}$ but they require data about participant locations (home, work, school) that can be difficult to obtain.

In this review, most studies with adult participants focused on retailer density in egocentric neighbourhoods, using street network buffers around home or constructed activity spaces, while several opted for administrative measures per land area, particularly in census tract and residential ZIP codes. In adolescent studies, density measures within egocentric circular buffers near schools and in administrative units relative to population count were more commonly employed. These measures were generally consistent with recommendations of the PhenX Toolkit for tobacco regulatory research, ${ }^{24}$ and similar to the findings of the recent methodological review, ${ }^{21}$ none provided a clear advantage in revealing associations. Retailer proximity for both populations was commonly measured as the shortest road network distance or straight-line distance to the nearest retailer. Less common measures that were not included in the PhenX Toolkit, such as travel time by car, or presence of at least one retailer within a certain distance, were used, but did not show a significant advantage in revealing associations.

Differences between local or national tobacco policies across study settings may further limit comparability and partially explain null findings. Compliance with youth access laws, for example, may mitigate/moderate the relationship between retail density and adolescent smoking. ${ }^{33} 39$ Smoke-free air policies have also been shown to moderate this association. ${ }^{53}$ However, with the exception of a few studies, ${ }^{43} 4753$ the effects of such policies have not been accounted for. Another moderating influence may be point-of-sale advertising and display bans, which are effective in reducing smoking in adolescents $^{57} 58$ and adults ${ }^{59}$ and therefore are likely to be another moderating influence. Notably, studies from Quebec, Canada and Finland, where point-of-sale advertising restrictions have long been in place, still found retailer density/ proximity associated with lower adult cessation rates, ${ }^{28} 3435$ suggesting that retail availability affects smoking behaviour independent of advertising exposure. Finally, given that racially diverse and socioeconomically disadvantaged neighbourhoods have significantly higher density of tobacco retailers, ${ }^{3} 4346061$ the relationship between retailer density and individual smoking behaviour is likely modified by neighbourhood socioeconomic status (SES), ${ }^{46}$ which many studies did not address. Inconsistent findings may also be attributed to the different operational definitions of this concept across studies. Future research should also include spatial measures that better capture racial residential disparities, such as historical redlining. ${ }^{62}$

Increasingly, jurisdictions are implementing policies to reduce the spatial availability of tobacco products. ${ }^{63}$ Evidence is beginning to emerge regarding their impact on tobacco use, ${ }^{65} 66$ although it may take years before changes may be seen at the population level. ${ }^{67}$ Simulation models examining the impact of various retail restrictions estimate reduced smoking prevalence and health benefits. ${ }^{58-70}$ However, evidence suggests that there is no standard approach to retailer reduction policies, and their effects may vary across different settings. ${ }^{6}$

Overall, this review supports the view that reducing tobacco retailer density may help reduce adult and youth smoking prevalence. To our knowledge, this is the first review to consider the relationship between tobacco retailer availability/accessibility in different geographical settings and cigarette and e-cigarette use by adolescents and adults. An important strength of this review is that it considered multiple tobacco use outcomes and compared various measures of density and proximity. However, the review has several limitations. Since the emphasis of this scoping review was to provide a comprehensive overview of the current literature regardless of the standard of evidence, the critical assessment of the quality of included studies was not performed. This limits our ability to provide concrete guidance to inform policymaking. Further, most studies were cross-sectional, making it difficult to distinguish whether increased retail density/proximity increases the odds of smoking, or whether tobacco retailers are locating their businesses in response to high market demand. Nevertheless, evidence from longitudinal studies suggests a causal effect of living in areas with densely distributed tobacco retailers or in their close proximity and decreased adult cessation. ${ }^{34} 35$ Finally, while some studies had a fixed neighbourhood buffer zone to measure retailer density, others chose increasing intervals of buffers. In such studies, we reported a buffer size closest to the one across the included studies for the purpose of comparability, which may have biased the results. Future research should consider sensitivity analysis regarding buffer sizes used across studies, perhaps separately for urban and rural areas. A uniform grid unit method for geospatial distribution of tobacco retailers, with larger grid units in rural versus urban areas, is recommended. ${ }^{71}$ Tobacco retail accessibility may play an important role in individual smoking behaviour, particularly in rural areas, ${ }^{72}$ but remains largely unexplored. Specific measures of retail accessibility, such as travel time by car, should be considered in the PhenX Toolkit of recommended measures for tobacco regulatory research.

\section{CONCLUSION}

This scoping review finds some evidence of an association between tobacco retailer availability and smoking outcomes in youth and adults. More research is needed, particularly of longitudinal design, with representative samples, uniform measures of exposure and outcome variables, and consistent inclusion of major individual and area-level characteristics, such as racial diversity and neighbourhood SES. Quasi-experimental beforeafter studies are also needed to fill the gap in evidence regarding causality between retailer density/proximity and outcomes in youth and adults. Studies on the risk of cigarette initiation and tobacco retailer availability are particularly scarce and should be the focus of future research. Finally, studies examining associations between retailer availability and e-cigarette use are scarce and further research is warranted. 


\section{What this paper adds}

- Limiting tobacco retail availability may be an effective tobacco control strategy to reduce smoking and improve public health. Evidence on the associations between tobacco retailer density/proximity and cigarette/e-cigarette use is mixed and inconsistencies in measures of retailer exposure across studies have been reported. There was a need for a comprehensive literature review to summarise the existing evidence for both youth and adults and highlight the methodological gaps.

- This review suggests that tobacco retailer density, but not proximity, may be a contributing factor in promoting smoking among youth and adults. In particular, future tobacco control policies limiting retailer exposure in residential areas may be successful in reducing smoking, while reducing tobacco retailer availability around schools may not be as effective. Research on e-cigarette use and density/proximity of ecigarette retailers is surprisingly scarce, given their popularity among youth. There is a need for more research with representative samples, uniform measures of exposure and outcome variables, and consistent control for major area-level characteristics, such as racial diversity and neighbourhood disparity.

Contributors DTL and NT came up with the idea for the article. NT performed the literature search, wrote the article, has access to all data and is the guarantor for the finished article. PAM, DTL and LH made substantial contributions to the conception of the work and the interpretation of findings. All authors participated in the review and final approval of the manuscript.

Funding This research was funded by a grant from the National Cancer Institute (1R01-CA229238, PI: Ruth E Malone).

Disclaimer The opinions expressed in this article are the authors' own and do not reflect the views of the National Institutes of Health, the Department of Health and Human Services, or the US government.

Competing interests None declared.

Patient consent for publication Not required.

Provenance and peer review Not commissioned; externally peer reviewed.

Open access This is an open access article distributed in accordance with the Creative Commons Attribution Non Commercial (CC BY-NC 4.0) license, which permits others to distribute, remix, adapt, build upon this work non-commercially, and license their derivative works on different terms, provided the original work is properly cited, appropriate credit is given, any changes made indicated, and the use is non-commercial. See: http://creativecommons.org/licenses/by-nc/4.0/.

\section{ORCID iDs}

Nargiz Travis http://orcid.org/0000-0001-6305-8259

David T Levy http://orcid.org/0000-0001-5280-3612

Patricia A McDaniel http://orcid.org/0000-0002-2750-4124

Lisa Henriksen http://orcid.org/0000-0002-9288-3562

\section{REFERENCES}

1 U.S. Department of Health and Human Services, The Health Consequences of Smoking: 50Years of Progress. A report of the Surgeon General. Atlanta, GA US Department of Health and Human Services, Centers for Disease Control and Prevention, National Center for Chronic Disease Prevention and Health Promotion, Office on Smoking and Health; 2014 [Accessed Feb 2020].

2 Corey CG, Holder-Hayes E, Nguyen AB, et al. Us adult cigar smoking patterns, purchasing behaviors, and reasons for use according to cigar type: findings from the population assessment of tobacco and health (path) study, 2013-2014. Nicotine Tob Res 2018;20:1457-66.

3 Marashi-Pour S, Cretikos M, Lyons C, et al. The association between the density of retail tobacco outlets, individual smoking status, neighbourhood socioeconomic status and school locations in New South Wales, Australia. Spat Spatiotemporal Epidemiol $2015 ; 12: 1-7$
4 Novak SP, Reardon SF, Raudenbush SW, et al. Retail tobacco outlet density and youth cigarette smoking: a propensity-modeling approach. Am J Public Health 2006;96:670-6.

5 Pearson AL, Cleghorn CL, van der Deen FS, et al. Tobacco retail outlet restrictions: health and cost impacts from multistate life-table modelling in a national population. Tob Control 2016:tobaccocontrol-2015-052846.

6 Luke DA, Hammond RA, Combs T, et al. Tobacco town: computational modeling of policy options to reduce tobacco Retailer density. Am J Public Health 2017; 107:740-6

7 Leatherdale ST, Strath JM. Tobacco retailer density surrounding schools and cigarette access behaviors among underage smoking students. Ann Behav Med 2007;33:105-11.

8 Schleicher NC, Johnson TO, Fortmann SP, et al. Tobacco outlet density near home and school: associations with smoking and norms among US teens. Prev Med 2016;91:287-93.

9 Loomis BR, Kim AE, Busey AH, et al. The density of tobacco retailers and its association with attitudes toward smoking, exposure to point-of-sale tobacco advertising, cigarette purchasing, and smoking among New York youth. Prev Med 2012;55:468-74.

10 Wood L, Gazey A, Murray K, et al. Unplanned purchasing of tobacco products: beyond point of sale display. Health Promot J Austr 2020;31:140-4.

11 Johns M, Sacks R, Rane M, et al. Exposure to tobacco retail outlets and smoking initiation among New York City adolescents. J Urban Health 2013;90:1091-101.

12 Robertson L, McGee R, Marsh L, et al. A systematic review on the impact of point-ofsale tobacco promotion on smoking. Nicotine Tob Res 2015;17:2-17.

13 Siahpush M, Shaikh RA, Cummings KM, et al. The association of point-of-sale cigarette marketing with cravings to smoke: results from a cross-sectional populationbased study. Tob Control 2016;25:402-5.

14 Chaiton MO, Mecredy GC, Cohen JE, et al. Tobacco retail outlets and vulnerable populations in Ontario, Canada. Int J Environ Res Public Health 2013;10:7299-309.

15 Hawkins SS, Bach N, Baum CF. Impact of tobacco control policies on adolescent smoking. J Adolesc Health 2016;58:679-85.

16 Lovato C, Watts A, Brown KS, et al. School and community predictors of smoking: a longitudinal study of Canadian high schools. Am J Public Health 2013;103:362-8.

17 Finan LJ, Lipperman-Kreda S, Abadi M, et al. Tobacco outlet density and adolescents' cigarette smoking: a meta-analysis. Tob Control 2019;28:27-33.

18 Gwon SH, DeGuzman PB, Kulbok PA, et al. Density and proximity of licensed tobacco Retailers and adolescent smoking. J Sch Nurs 2017;33:18-29.

19 Marsh L, Vaneckova P, Robertson L, et al. Association between density and proximity of tobacco retail outlets with smoking: a systematic review of youth studies. Health Place 2021;67:102275.

20 Nuyts PAW, Davies LEM, Kunst AE. The association between tobacco outlet density and smoking among young people: a systematic methodological review. Nicotine Tob Res 2019:ntz153.

21 Valiente $\mathrm{R}$, Escobar F, Urtasun $\mathrm{M}$, et al. Tobacco retail environment and smoking: a systematic review of geographic exposure measures and implications for future studies. Nicotine Tob Res 2020. doi:10.1093/ntr/ntaa223. [Epub ahead of print: 06 Nov 2020].

22 Cullen KA, Gentzke AS, Sawdey MD, et al. E-Cigarette use among youth in the United States, 2019. JAMA 2019:322:2095.

23 Gentzke AS, Creamer M, Cullen KA, et al. Vital Signs: Tobacco Product Use Among Middle and High School Students - United States, 2011-2018. MMWR Morb Mortal Wkly Rep 2019;68:157-64.

24 Ribisl KM, Chaloupka FJ, Kirchner TR, et al. PhenX: vector measures for tobacco regulatory research. Tob Control 2020;29:s27-34.

25 Chaiton MO, Mecredy G, Cohen J. Tobacco retail availability and risk of relapse among smokers who make a quit attempt: a population-based cohort study. Tob Control 2018;27:163-9.

26 Fleischer NL, Lozano P, Wu Y-H. Disentangling the roles of point-of-sale ban, tobacco retailer density and proximity on cessation and relapse among a cohort of smokers: findings from ITC Canada survey. Tob Control 2019;28:81-7.

27 Kirst M, Chaiton M, O'Campo P. Tobacco outlet density, neighbourhood stressors and smoking prevalence in Toronto, Canada. Health Place 2019;58:102171.

28 Shareck M, Datta GD, Vallée J. Is smoking cessation in young adults associated with tobacco retailer availability in their activity space? Nicotine Tob Res 2018.

29 Shareck M, Kestens Y, Vallée J, et al. The added value of accounting for activity space when examining the association between tobacco retailer availability and smoking among young adults. Tob Control 2016;25:406-12.

30 Chan WC, Leatherdale ST. Tobacco retailer density surrounding schools and youth smoking behaviour: a multi-level analysis. Tob Induc Dis 2011;9:9.

31 Cole AG, Aleyan S, Leatherdale ST. Exploring the association between e-cigarette retailer proximity and density to schools and youth e-cigarette use. Prev Med Rep 2019;15:100912

32 Pearce J, Hiscock R, Moon G, et al. The neighbourhood effects of geographical access to tobacco retailers on individual smoking behaviour. J Epidemiol Community Health 2009;63:69-77.

33 Marsh L, Ajmal A, McGee R, et al. Tobacco retail outlet density and risk of youth smoking in New Zealand. Tob Control 2016;25:e71-4. 
34 Pulakka A, Halonen JI, Kawachi I, et al. Association between distance from home to tobacco outlet and smoking cessation and relapse. JAMA Intern Med 2016;176:1512-9.

35 Halonen Jl, Kivimäki M, Kouvonen A, et al. Proximity to a tobacco store and smoking cessation: a cohort study. Tob Control 2014;23:146-51.

36 Barnes R, Foster SA, Pereira G, et al. Is neighbourhood access to tobacco outlets related to smoking behaviour and tobacco-related health outcomes and hospital admissions? Prev Med 2016;88:218-23.

37 Scully M, McCarthy M, Zacher M, et al. Density of tobacco retail outlets near schools and smoking behaviour among secondary school students. Aust N Z J Public Health 2013;37:574-8

38 Pearce J, Rind E, Shortt N, et al. Tobacco retail environments and social inequalities in individual-level smoking and cessation among Scottish adults. Nicotine Tob Res 2016;18:138-46.

39 Shortt NK, Tisch C, Pearce J, et al. The density of tobacco retailers in home and school environments and relationship with adolescent smoking behaviours in Scotland. Tob Control 2016;25:75-82.

40 Bostean G, Crespi CM, Vorapharuek P, et al. E-Cigarette use among students and e-cigarette specialty retailer presence near schools. Health Place 2016;42:129-36.

41 Giovenco DP, Casseus M, Duncan DT, et al. Association between electronic cigarette marketing near schools and e-cigarette use among youth. J Adolesc Health 2016;59:627-34.

42 Trapl E, Anesetti-Rothermel A, Pike Moore S. Association between school-based tobacco retailer exposures and young adolescent cigarette, cigar and e-cigarette use. Tob Control 2020.

43 Cantrell J, Pearson JL, Anesetti-Rothermel A, et al. Tobacco retail outlet density and young adult tobacco initiation. Nicotine Tob Res 2016;18:130-7.

44 Abdel Magid HS, Halpern-Felsher B, Ling PM, et al. Tobacco retail density and initiation of alternative tobacco product use among teens. J Adolesc Health 2020;66:30447-30441.

45 Cantrell J, Anesetti-Rothermel A, Pearson JL, et al. The impact of the tobacco retail outlet environment on adult cessation and differences by neighborhood poverty. Addiction 2015;110:152-61.

46 Chuang Y-C, Cubbin C, Ahn D, et al. Effects of neighbourhood socioeconomic status and convenience store concentration on individual level smoking. J Epidemiol Community Health 2005;59:568-73

47 Adams ML, Jason LA, Pokorny $\mathrm{S}$, et al. Exploration of the link between tobacco retailers in school neighborhoods and student smoking. J Sch Health 2013;83:112-8.

48 Lipperman-Kreda S, Finan LJ, Kowitt SD, et al. Youth daily exposure to tobacco outlets and cigarette smoking behaviors: does exposure within activity space matter? Addiction 2020:115:1728-35

49 Lipperman-Kreda S, Mair C, Grube JW, et al. Density and proximity of tobacco outlets to homes and schools: relations with youth cigarette smoking. Prev SCi 2014;15:738-44.

50 McCarthy WJ, Mistry R, Lu Y, et al. Density of tobacco retailers near schools: effects on tobacco use among students. Am J Public Health 2009;99:2006-13.

51 Kirchner TR, Anesetti-Rothermel A, Bennett M, et al. Tobacco outlet density and converted versus native non-daily cigarette use in a national US sample. Tob Control 2017;26:85-91.

52 Adachi-Mejia AM, Carlos HA, Berke EM, et al. A comparison of individual versus community influences on youth smoking behaviours: a cross-sectional observational study. BMJ Open 2012;2. doi:10.1136/bmjopen-2011-000767. [Epub ahead of print: 01 Sep 2012].
53 Lipperman-Kreda S, Grube JW, Friend KB. Local tobacco policy and tobacco outlet density: associations with youth smoking. J Adolesc Health 2012;50:547-52.

54 Lipperman-Kreda S, Grube JW, Friend KB, et al. Tobacco outlet density, retailer cigarette sales without ID checks and enforcement of underage tobacco laws: associations with youths' cigarette smoking and beliefs. Addiction 2016;111:525-32.

55 Pokorny SB, Jason LA, Schoeny ME. The relation of retail tobacco availability to initiation and continued smoking. J Clin Child Adolesc Psychol 2003;32:193-204.

56 Duncan DT, Kawachi I, Subramanian SV, et al. Examination of how neighborhood definition influences measurements of youths' access to tobacco retailers: a methodological note on spatial misclassification. Am J Epidemiol 2014;179:373-81

57 Haw S, Currie D, Eadie D, et al. The impact of the point-of-sale tobacco display ban on young people in Scotland: before-and-after study. Public Health Res 2020;8:1-118.

58 Edwards R, Ajmal A, Healey B, et al. Impact of removing point-of-sale tobacco displays: data from a new Zealand youth survey. Tob Control 2017;26:392-8.

59 He Y, Shang C, Huang J, et al. Global evidence on the effect of point-of-sale display bans on smoking prevalence. Tob Control 2018;27:e98-104.

60 Lee JGL, Sun DL, Schleicher NM, et al. Inequalities in tobacco outlet density by race, ethnicity and socioeconomic status, 2012, USA: results from the ASPiRE study. $J$ Epidemiol Community Health 2017;71:487-92.

61 Siegel SD, Brooks MM, Gbadebo BM, et al. Using Geospatial analyses of linked electronic health records and tobacco outlet data to address the social determinants of smoking. Prev Chronic Dis 2019;16:E152

62 Schwartz E, Onnen N, Craigmile PF, et al. The legacy of redlining: associations between historical neighborhood mapping and contemporary tobacco retailer density in Ohio. Health Place 2021;68:102529.

63 Vyas P, Sturrock H, Ling PM. Examining the role of a retail density ordinance in reducing concentration of tobacco retailers. Spat Spatiotemporal Epidemiol 2020;32:100307.

64 Lawman HG, Henry KA, Scheeres A, et al. Tobacco retail licensing and density 3 years after license regulations in Philadelphia, Pennsylvania (2012-2019). Am J Public Health 2020;110:547-53.

65 Ali FRM, Neff L, Wang X, et al. Tobacco-Free pharmacies and U.S. adult smoking behavior: evidence from CVS health's removal of tobacco sales. Am J Prev Med 2020;58:41-9.

66 Polinski JM, Howell B, Gagnon MA, et al. Impact of CVS pharmacy's discontinuance of tobacco sales on cigarette purchasing (2012-2014). Am J Public Health 2017; 107:556-62.

67 Glasser AM, Roberts ME. Retailer density reduction approaches to tobacco control: a review. Health Place 2021;67:102342.

68 Robertson L, Marsh L. Estimating the effect of a potential policy to restrict tobacco retail availability in New Zealand. Tob Control 2019;28:466-8.

69 Pearson AL, van der Deen FS, Wilson N, et al. Theoretical impacts of a range of major tobacco retail outlet reduction interventions: modelling results in a country with a smoke-free nation goal. Tob Control 2015;24:e32-8.

70 Marsh L, Doscher C, Cameron C, et al. How would the tobacco retail landscape change if tobacco was only sold through liquor stores, petrol stations or pharmacies? Aust N Z J Public Health 2020;44:34-9.

71 Lipton $\mathrm{R}$, Banerjee $A$. The geography of chronic obstructive pulmonary disease across time: California in 1993 and 1999. Int J Med Sci 2007;4:179-89.

72 Doogan NJ, Cooper S, Quisenberry AJ, et al. The role of travel distance and price promotions in tobacco product purchase quantity. Health Place 2018;51:151-7. 